Bond University

Research Repository

\title{
Trait impulsivity predicts D-KEFS Tower Test performance in university students
}

Lyvers, Michael; Basch, Vanessa; Duff, Helen; Edwards, Mark S.

Published in:

Applied Neuropsychology: Adult

DOI:

$10.1080 / 23279095.2013 .850693$

\section{Licence:}

CC BY-NC-ND

Link to output in Bond University research repository.

Recommended citation(APA):

Lyvers, M., Basch, V., Duff, H., \& Edwards, M. S. (2015). Trait impulsivity predicts D-KEFS Tower Test performance in university students. Applied Neuropsychology: Adult, 22(2), 88-93.

https://doi.org/10.1080/23279095.2013.850693

\footnotetext{
General rights

Copyright and moral rights for the publications made accessible in the public portal are retained by the authors and/or other copyright owners and it is a condition of accessing publications that users recognise and abide by the legal requirements associated with these rights.
}

For more information, or if you believe that this document breaches copyright, please contact the Bond University research repository coordinator. 
Trait Impulsivity Predicts D-KEFS Tower Test Performance in University Students

This research was part of a larger study funded by a grant from the Foundation for Alcohol Research and Education Limited. There were no conflicts of interest. 


\begin{abstract}
The present study examined a widely used self-report index of trait impulsiveness in relation to performance on a well-known neuropsychological executive function test in 70 university undergraduate students (50 females, 20 males) aged 18-24 years. Participants completed the Barratt Impulsiveness Scale (BIS-11) and the Frontal Systems Behavior Scale (FrSBe), after which they performed the Tower Test of the Delis-Kaplan Executive Function System (DKEFS). Hierarchical linear regression showed that after controlling for gender, current alcohol consumption, age at onset of weekly alcohol use, and FrSBe scores, BIS-11 significantly predicted Tower Test Achievement scores, $\beta=-.44, p<.01$. The results indicate that self-reported impulsiveness is associated with poorer executive cognitive performance even in a sample likely to be characterized by relatively high general cognitive functioning (i.e., university students) and support the role of inhibition as a key aspect of executive task performance. Elevated scores on BIS-11 and FrSBe are known to be linked to risky drinking in young adults as confirmed in this sample, however only BIS-11 predicted Tower Test performance.
\end{abstract}

Keywords: executive function, impulsiveness, frontal lobes, personality, cognition 
Trait Impulsivity Predicts D-KEFS Tower Test Performance in University Students

Although a number of self-report measures purporting to assess everyday executive functioning have been developed to date, the relationship of self-report indices to actual performance on executive function tasks has been inconsistent at best. A recent review (Toplak, West \& Stanovich, 2013) of 20 published studies of such relationships found that self-report and task performance measures were significantly correlated in only 68 of 286 correlations (i.e., 24\%) with a median correlation of only .19. Toplak et al. concluded that different aspects of executive function may be tapped by self-report versus task performance measures and thus these two sources of information about executive function should not be regarded as interchangeable.

A recent theoretical paper by Miyake and Friedman (2012) concluded that the ability to inhibit impulses is a key aspect of executive function as measured by executive function tasks. As high impulsivity is associated with a variety of clinical conditions and problematic behaviours, self-report scales have been developed to measure this trait. One of the most widely used self-report measures of impulsivity is the Barratt Impulsiveness Scale (BIS-11; Patton, Stanford \& Barratt, 1995). Scores on this measure have been reported to be significantly elevated in various clinical samples, and in non-clinical samples BIS-11 scores have been reported to negatively correlate with performance of go/no-go, Stroop interference and antisaccade tests as well as brain imaging signs of prefrontal cortex activation (Asahi, Okamoto, Okada, Yamawaki \& Yokota, 2004; Enticott, Ogloff \& Bradshaw, 2006; Spinella, 2004). Recent work in university student and community samples (Lyvers, Duff \& Hasking, 2011: Lyvers, Duff, Basch \& Edwards, 2012) also indicates that BIS-11 scores tend to be highly positively correlated with scores on the Frontal Systems Behavior Scale (FrSBe; Grace \& Malloy, 2001), a self-report index of executive function designed to assess behaviour problems resulting from prefrontal cortical injury. Lyvers et al. $(2011,2012)$ further found 
that in young adults BIS-11 scores were highly positively correlated with scores on the Alcohol Use Disorders Identification Test (AUDIT; Babor, de la Fuente, Saunders, \& Grant, 1992), and were negatively correlated with drinking onset age such that earlier onset of weekly drinking was associated with higher BIS-11 scores. Evidence thus suggests that high impulsivity as measured by the BIS-11 may be related to prefrontal cortex and executive dysfunction even in non-clinical samples, and may constitute a significant risk factor for problematic alcohol use (Dawe, Gullo \& Loxton, 2004; Lyvers et al., 2011, 2012).

Despite the widespread use of the BIS-11 as a trait measure of impulsiveness - and the importance of inhibition as a key component of executive task performance (Miyake \& Friedman, 2012) - the relationship of BIS-11 scores to executive task performance has received scant attention. Only three studies in Toplak et al.'s (2013) review had examined the relationship between impulsivity self-ratings and executive task performance measures, with only $19 \%$ of correlations significant and a median correlation of .25 . Of the three studies only one examined the relationship between the BIS-11 measure of impulsivity and task performance, reporting that BIS-11 was highly positively correlated $(r=.55)$ with an index of the Stroop task interference effect (Enticott et al., 2006). The relatively strong reported relationship between BIS-11 and Stroop task performance suggests that BIS-11 might be unusual among self-report measures as a predictor of executive task performance. Further, given its strong correlation with FrSBe scores (Lyvers et al., 2011, 2012) the ability of the latter to predict executive task performance also merits examination as well.

The present study examined BIS-11, FrSBe, AUDIT, and drinking onset age in relation to university students' performance of a well-known and widely used executive task, the Tower Test of the Delis-Kaplan Executive Function System (D-KEFS; Delis, Kaplan \& Kramer, 2001). The Tower Test was chosen because inhibition of impulsive or perseverative response tendencies was described by Delis et al. as one of the crucial functions assessed by 
this task. Tower Test performance is significantly impaired in patients with damage to the prefrontal cortex (Yochim, Baldo, Kane \& Delis, 2009), a brain region where task-related activation has been found to negatively correlate with BIS-11 scores (Asahi et al., 2004). The present study asked whether BIS-11 and/or FrSBe scores would be able to predict performance on the Tower Test in a hierarchical regression even in a healthy university student sample after controlling for alcohol use history and current drinking levels, as chronic heavy drinking is known to adversely impact performance on neuropsychological tests of executive cognitive functioning in older adults (Lyvers, 2000) and acute alcohol intoxication has similar effects on such performance in young adults (Lyvers \& Tobias-Webb, 2010).

\section{Method}

\section{Participants}

Participants were recruited from the Bond University undergraduate student research participant pool via a sign-up sheet. Full informed consent was obtained prior to participation, and experimental credit toward an introductory psychology or marketing subject was the incentive. Participants were screened for blood alcohol on the day of testing using a breathalyser, as alcohol intoxication impairs executive cognitive functioning (Lyvers \& Tobias-Webb, 2010); two volunteers were excluded for this. Further exclusion criteria of no neurological or psychiatric illness, and no previously incurred brain trauma, resulted in four more participants being excluded. Finally, four multivariate outliers were identified via Mahalanobis distance using Boxplots and removed from the dataset. The final sample was thus comprised of 70 participants (50 females, 20 males) aged 18 to 24 years $(M=19.77 ; S D$ $=1.32$ ) and all were native English speakers. The sample reported very low rates of illicit drug use and smoking, however hazardous alcohol consumption was indicated by the high mean AUDIT score $(M=11.53, S D=6.34)$ which is common in university student samples, most likely due to the social context of university life (Lyvers, Czerczyk, Follent \& Lodge, 
2009; Lyvers et al., 2011, 2012). All participants reported they were unfamiliar with the Tower Test, and all were at least occasional alcohol users (the legal drinking age in Australia is 18 years).

\section{Materials}

Barratt Impulsiveness Scale (BIS-11; Patton et al., 1995). The BIS-11 is designed to assess trait impulsivity. The scale consists of 30 items covering the domains of nonplanning impulsivity (e.g., "I plan tasks carefully" - reverse scored item); motor impulsivity or acting on the spur of the moment (e.g., "I do things without thinking," "I buy things on impulse"); and attentional impulsivity or the inability to focus on the task at hand (e.g., "I don't pay attention”). Items on the BIS-11 are rated on a four-point Likert scale, (0) Rarely, (1) Occasionally, (2) Often, and (3) Almost Always/Always. A reverse scoring procedure is used for 11 items. After reverse scoring is applied, a total impulsivity score is established by summing the scores on all 30 items. The alpha reliability coefficient was .84 in the present sample.

\section{Delis-Kaplan Executive Function System (D-KEFS) - Tower Test (Delis et al.,}

2001). The D-KEFS Tower Test is a measure of executive functions, assessing rule learning, spatial planning, inhibition of perseverative and impulsive responding, and the ability to establish and maintain instructional set. The D-KEFS Tower Test consists of a board with three vertical pegs and five disks that vary in size. There are nine items in the D-KEFS Tower Test; for each item the disks are placed in a predetermined starting position and a picture of the tower to be built is displayed to the examinee. To complete each item the examinee is asked to build the target tower in the fewest number of moves possible by moving the disks across the three pegs. The examinee is required to follow two rules: 1) move only one disk at a time using only one hand to move each disk, and 2) do not place a larger disk on top of a smaller disk. Difficulty of completing the task increases with each item, and therefore the 
minimum number of moves required to complete each target tower varies from one move (Item 1) to 26 moves (Item 9). A Total Achievement Score is calculated by summing the total number of moves for all items administered, and can range from 0 (all items failed) to 30; this constitutes an overall measure of performance on the D-KEFS Tower Test that is significantly lower in patients with focal lesions of the prefrontal cortex than in controls (Yochim et al., 2009). The D-KEFS Tower Test has been used in Australian research despite having been normed on a U.S. sample; in such instances it is common practice to cautiously assume equivalence between Australia and the U.S. in this regard (Lyvers, Tobias-Webb \& Edwards, 2012; Remine, Care \& Brown, 2008).Shunk, Davis and Dean’s (2006) review concluded that the test is psychometrically sound, however low reliability statistics are acknowledged as a potential limitation.

Frontal Systems Behaviour Scale (FrSBe; Grace \& Malloy, 2001). The FrSBe consists of 46 items designed to assess behavioural syndromes associated with damage to the prefrontal cortex. The FrSBe has three subscales, Apathy (14 items), Disinhibition (15 items), and Executive Dysfunction (17 items), which are considered to reflect functioning of the anterior cingulate, orbitofrontal cortex, and dorsolateral prefrontal cortex, respectively. A total FrSBe score is obtained by summing the subscale scores and was used in this study. For the purposes of this study, the FrSBe was adapted as in other recent research such that responses were based on the participant's current situation (Lyvers et al., 2009; Spinella, 2003; Verdejo-Garcia et al., 2006) rather than before versus after brain injury. Administration time of the FrSBe is approximately 10 to 15 minutes. FrSBe items are answered by choosing one of five anchors on a Likert scale, (1) Almost Never, (2) Seldom, (3) Sometimes, (4) Frequently, and (5) Almost Always. A reverse scoring procedure is implemented on items 33 to 46 of the FrSBe. Higher total FrSBe scores suggest a greater degree of impairment. In the present sample the alpha reliability coefficient was .84 . 
Alcohol Use Disorders Identification Test (AUDIT; Babor et al., 1992). The

AUDIT is a self-report screening measure designed to assess alcohol-related risk in clinical and non-clinical populations (Reinert \& Allen, 2002). The measure consists of 10 items based on three domains of alcohol use. Items 1 to 3 assess alcohol consumption (e.g. "How often do you have a drink containing alcohol?"), items 4 to 6 assess alcohol dependence (e.g. "How often during the last year have you failed to do what was normally expected from you because of drinking?"), and items 7 to 10 assess alcohol related harm (e.g. "Have you or someone else been injured as a result of your drinking?”). The AUDIT takes approximately two minutes to administer, with each item rated on a scale of 0 to 4 . The total score is established by summing the values for each item, with a maximum score of 40 . A total score of 0 to 7 indicates low risk alcohol consumption, whereas scores of 8 to 15 indicate hazardous drinking and scores of 16 and above suggest harmful drinking (Babor, HigginsBiddle, Saunders, \& Monteiro, 2001). The alpha reliability coefficient was .83 in the present sample.

Demographic Questionnaire. A demographic questionnaire was completed by all participants, and included questions regarding age, age at onset of weekly drinking (AOD), gender, illicit drug use, and smoking. Further questions pertaining to alcohol consumed on day of testing, previous brain trauma, and current neurological or psychiatric condition were included for screening purposes.

\section{Procedure}

The Bond University Human Research Ethics Committee (BUHREC) provided ethical clearance for the study. Undergraduate students were then recruited through sign-up sheets attached to an explanatory statement which described the nature of the research and exclusion criteria. Participants were tested individually in a laboratory room on campus and in return received course credit towards an introductory psychology or marketing subject. 
Upon arrival to the testing room, participants were seated at a computer and read an online explanatory statement, after which they provided online consent to participate. Participants were then required to complete the self-report questionnaires using a commercial on-line survey program, Survey Monkey. Presentation order of the questionnaires was the same for all participants. Following completion of the questionnaires, the D-KEFS Tower Test was manually administered by the researcher. The participant was seated at a table and instructions were provided in accordance with the D-KEFS Tower Test Administration Manual (Delis et al., 2001). A wooden tower base was placed on the table in front of the participant along with the D-KEFS stimulus book. The ending position illustration of Item 1 was shown to the participant, and five blue disks were placed next to the wooden base. Participants were instructed to use the pieces to build a tower that looked like the picture in the stimulus book, using the fewest number of moves possible. Participants were informed of the two rules they needed to follow, in accordance with the standardised instructions. The examiner began each item by presenting disks on the pegs in a predetermined starting position and displayed a picture that showed the ending position of the disks to the participant. Each item was administered until all nine items were completed, or the participant failed three consecutive items at which point the test was stopped. A stopwatch was used to measure the time it took the participant to complete each item, as there was a discontinue time limit for each item. The participant's scores for the D-KEFS Tower Test were recorded on the Tower Test Response Sheet. Each tower correctly built within the set time limit was scored 1 point, with bonus marks allocated for a tower built in the minimum moves possible. After completion of testing the participant was thanked and provided with a credit slip giving them one credit point for their undergraduate psychology or marketing subject.

\section{Results}

Intercorrelations were calculated among the continuous variables, which were related 
in expected ways. As shown in Table 1, BIS-11 total impulsivity scores were significantly negatively correlated with Tower Test Total Achievement scores as predicted. Further, BIS11 scores were significantly positively correlated with total FrSBe scores and AUDIT scores as in previous work (Lyvers et al., 2011, 2012), and were significantly negatively correlated with AOD. Interestingly, AOD was significantly positively correlated with Tower Test Total Achievement scores (see Table 1), such that later onset age of weekly drinking was associated with better Tower Test performance as well as lower impulsivity scores.

Hierarchical linear regression was conducted on Tower Test Total Achievement scores. Gender, AUDIT and AOD were entered at step 1, followed by FrSBe scores at step 2 and BIS-11 at step 3. Only the final model was significant, $R=.40, R^{2}=.16, F(5,64)=2.44$, $p=.04$. Table 2 shows the unstandardised regression coefficients (B), standardised regression coefficients $(\beta), t$ scores and the $R^{2}$ change for each predictor at each step. In the final model, BIS-11 was the only significant predictor, $p=.01$ (see Table 2).

\section{Discussion}

Present findings indicate that even among young adults with relatively high levels of cognitive functioning - i.e., university students - self-reported trait impulsiveness as measured by BIS-11 can predict performance on the D-KEFS Tower Test, a well-known neuropsychological test assessing executive function and one which is highly sensitive to prefrontal cortical injury (Yochim et al., 2009). By contrast, scores on a self-report measure designed to detect behavioural sequelae of prefrontal cortical injury in everyday life, the FrSBe, did not predict Tower Test performance in this sample, nor did the AOD and AUDIT indices of alcohol use. The failure to find a significant relationship of Tower Test performance to FrSBe scores in university students despite the significant relationships of both with BIS-11 may be because the FrSBe was designed to detect behavioural sequelae of brain injury whereas the BIS-11 was designed to measure a personality trait. The screening 
criteria for participation in this study included no history of brain trauma and no neurological or psychiatric illness, as well as a zero blood alcohol level. As in previous work, BIS-11 scores were significantly positively correlated with both AUDIT and FrSBe, and were significantly negatively correlated with AOD, consistent with the notion that trait impulsivity represents a risk factor for earlier and riskier drinking (Dawe et al., 2004; Lyvers et al., 2011, 2012).

The proportion of variance in Tower Test performance (as indexed by Total Achievement scores) that was explained by BIS-11 in the final step of the regression was small, only $9 \%$, thus other factors obviously contribute to performance on this test. Miyake and Friedman (2012) point out that all executive function tasks suffer from "task impurity" as a variety of non-executive-function processes are also required to perform them, and this clearly applies to the Tower Test as well. Nevertheless the finding that a self-report trait questionnaire can predict performance on a complex executive task such as the D-KEFS Tower Test is noteworthy and supports the validity of the BIS-11 as a trait measure that reflects a key aspect of executive function that varies significantly in the general population. Successful performance of the D-KEFS Tower Test requires attention, planning, inhibition of impulsive responding, and the ability to establish and maintain a solution strategy; the BIS-11 asks the respondent to evaluate similar cognitive-behavioural processes in a self-reflexive manner and like the Tower Test appears to be related to prefrontal cortical functioning (Asahi et al., 2004; Spinella, 2004; Yochim et al., 2009). In the present study participants completed the BIS-11 prior to undertaking the Tower Test so their BIS-11 scores were not influenced by their performance on the Tower Test; rather, BIS-11 scores presumably reflected what the participants had often observed in themselves in their everyday lives. In the present investigation, such self-reported observations translated into actual performance outcomes on a novel and complex neuropsychological task. Whether performance of other such tasks from 
the D-KEFS battery (Davis, Pierson \& Finch, 2011) as well as other executive function tasks (Reynolds \& MacNeill Horton Jr., 2008) can be predicted from responses on a self-report scale such as the BIS-11 remains an open question that should be addressed.

The recent review by Toplak et al. (2013) emphasised the rarity of such a finding, and concluded that self-report and task indices measure different aspects of executive function - a multifaceted construct - and should not be regarded as interchangeable. The present findings for the FrSBe are consistent with that conclusion, but present results also suggest that the BIS-11 may be unusual among self-report measures in terms of its relevance to outcomes on at least some neuropsychological tests of executive function, given its significant relationship to Tower Test performance in the present study and to Stroop task performance previously (Enticott et al., 2006). Interestingly, Miyake and Friedman (2012) recently concluded that, of three key facets of executive function, inhibition is the one most strongly related to executive task performance in general. Given the paucity of studies of self-rated impulsiveness in relation to executive task performance as reported by Toplak et al., and the significant role of impulsivity in a variety of problematic behaviors (Lyvers et al., 2011, 2012; Toplak et al.), further research on these relationships appears warranted. 


\section{References}

Asahi, S., Okamoto, Y., Okada, G., Yamawaki, S., \& Yokota, N. (2004). Negative correlation between right prefrontal activity during response inhibition and impulsiveness: A fMRI study. European Archives of Psychiatry and Clinical Neuroscience, 254(4), 245-251. doi:10.1007/s00406-004-0488-Z

Babor, T.F., de la Fuente, J.R., Saunders, J. and Grant, M. (1992). AUDIT: The alcohol use disorders identification test. Geneva: World Health Organization.

Babor, T. F., Higgins-Biddle, J. C., Saunders, J. B., \& Monteiro, M. G. (2001). The Alcohol Use Disorders Identification Test guidelines for use in primary care. . Geneva: World Health Organization (WHO).

Davis, A. S., Pierson, E. E., \& Finch, W. (2011). A canonical correlation analysis of intelligence and executive functioning. Applied Neuropsychology, 18, 61-68. doi:10.1080/09084282.2010.523392

Dawe, S., Gullo, M., J., \& Loxton, N. J. (2004). Reward drive and rash impulsiveness as dimensions of impulsivity: Implications for substance misuse. Addictive Behaviors, 29, 1389-1405. doi:10.1016/j.addbeh.2004.06.004

Delis, D. C., Kaplan, E., \& Kramer, J. H. (2001). Delis Kaplin Executive Function System: Examiner's manual. San Antonio, Texas: The Psychological Corporation. doi:10.1037/0894-4105.17.2.255

Enticott, P.G., Ogloff, J.R.P., \& Bradshaw, J.L. (2006). Associations between laboratory measures of executive inhibitory control and self-reported impulsivity. Personality and Individual Differences, 41, 285-294. doi:10.1016/j.paid.2006.01.011

Grace, J., \& Malloy, P. F. (2001). Frontal Systems Behavior Scale. Lutz, FL: PAR.

Lyvers, M. (2000). "Loss of control" in alcoholism and drug addiction: A neuroscientific interpretation. Experimental and Clinical Psychopharmacology, 8, 
225-249. doi:10.1037//1064-1297.8.2.225

Lyvers, M., Czerczyk, C., Follent, A., \& Lodge, P. (2009). Disinhibition and reward sensitivity in relation to alcohol consumption by university undergraduates. Addiction Research \& Theory, 17(6), 668-677. doi:10.3109/16066350802404158

Lyvers, M., Duff, H., \& Hasking, P. (2011). Risky alcohol use and age at onset of regular alcohol consumption in relation to frontal lobe indices, reward sensitivity, and rash impulsiveness. Addiction Research \& Theory, 19, 251-259.

doi:10.3109/16066359.16062010.16500751

Lyvers, M., Duff, H., Basch, V., \& Edwards, M. (2012). Influences of rash impulsiveness and reward sensitivity on risky drinking in university students: Evidence of mediation by frontal systems. Addictive Behaviors, 37, 940-946. doi:10.1016/j.addbeh.2012.03.028

Lyvers, M., \& Tobias-Webb, J. (2010). Effects of acute alcohol consumption on executive cognitive functioning in naturalistic settings. Addictive Behaviors, 35(11), 1021-1028. doi:10.1016/j.addbeh.2010.06.022

Lyvers, M., Tobias-Webb, J., \& Edwards, M. (2012). Neuropsychological Correlates of Risky Alcohol Use by Young Adults. Presented at the $75^{\text {th }}$ Annual Scientific Meeting of the Congress on Problems of Drug Dependence, Indian Wells California, 9-14 June 2012.

Miyake, A., \& Friedman, N.P. (2012). The nature and organization of individual differences in executive functions: Four general conclusions. Current Directions in Psychological Science, 21, 8-14. doi:10.1177/0963721411429458

Patton, J. H., Stanford, M. S., \& Barratt, E. S. (1995). Factor structure of the Barratt Impulsiveness Scale. Journal of Clinical Psychology, 51, 768 - 774. doi:10.1002/1097-4679(199511)51:6<768::AID-JCLP2270510607>3.0.CO;2-1

Reinert, D. F., \& Allen, J. P. (2002). The Alcohol Use Disorders Identification Test (AUDIT): 
A review of recent research. Alcoholism: Clinical and Experimental Research, 26(2), 272-279. doi:10.1111/j.1530-0277.2002.tb02534.x

Remine, M.D., Care, E., \& Brown, P.M. (2008). Language ability and verbal and nonverbal executive functioning in deaf students communicating in spoken English. Journal of Deaf Studies and Deaf Education, 13, 531-545. doi:10.1093/deafed/enn010

Reynolds, C. R., \& MacNeill Horton Jr., A. (2008). Assessing executive functions: A lifespan perspective. Psychology in the Schools, 45, 875-892. doi:10.1002/pits.20332

Shunk, A. W., Davis, A. S., \& Dean, R. S. (2006). Test review of the Delis-Kaplan Executive Function System. Applied Neuropsychology, 13, 275-279. doi:10.1207/s15324826an1304_9

Spinella, M. (2003). Relationship between drug use and prefrontal-associated traits. Addiction Biology, 8, 67-74. doi:10.1080/1355621031000069909

Spinella, M. (2004). Neurobehavioral correlates of impulsivity: Evidence of prefrontal involvement. International Journal of Neuroscience, 114, 95-104. doi:10.1080/00207450490249347

Toplak, M.E., West, R.F., \& Stanovich, K.E. (2013). Do performance-based measures and ratings of executive function assess the same construct? Journal of Child Psychology and Psychiatry, 54, 131-143. doi:10.1111/jcpp.12001

Verdejo-Garcia, A., Bechara, A., Recknor, E. C., \& Perez-Garcia, M. (2006). Executive dysfunction in substance dependent individuals during drug use and abstinence: An examination of the behavioral, cognitive, and emotional correlates of addiction. Journal of the International Neuropsychological Society, 12, 405-415. doi: $10.1017 / \mathrm{S} 1355617706060486$

Yochim, B.P., Baldo, J.V., Kane, K.D., \& Delis, D.C. (2009). D-KEFS Tower Test performance in patients with lateral prefrontal cortex lesions: The importance 
of error monitoring. Journal of Clinical and Experimental Neuropsychology, 31, 658-

663. doi:10.1080/13803390802448669 
Table 1.

Correlations among study variables: Age at Onset of weekly Drinking (AOD), Alcohol Use

Disorders Identification Test (AUDIT) score, Barratt Impulsiveness Scale (BIS-11) score, Frontal Systems Behavior Scale (FrSBe) score, and Tower Test Total Achievement score (Tower).

\begin{tabular}{rlccc}
\hline Variable & AOD & AUDIT & BIS-11 & FrSBe \\
\hline AUDIT & $-.40^{* * *}$ & & & \\
BIS-11 & $-.40^{* * *}$ & $.40 * * *$ & & \\
FrSBe & -.11 & .23 & $.63 * * *$ & \\
Tower & $.24 *$ & -.01 & $-.25 *$ & .04 \\
\hline & & & & \\
$* \begin{array}{l}*<.05 \\
*<* .001\end{array}$ & & & &
\end{tabular}


Table 2.

Predictors of Tower Test Total Achievement score: Gender, Age at Onset of weekly Drinking (AOD), Alcohol Use Disorders Identification Test (AUDIT) score, Frontal Systems Behavior Scale (FrSBe) score, and Barratt Impulsiveness Scale (BIS-11) score.

\begin{tabular}{|c|c|c|c|c|c|}
\hline & Variable & B & $\beta$ & $t$ & $R^{2}$ Change \\
\hline \multirow[t]{4}{*}{ Step 1} & (Constant) & 1.60 & & 0.41 & .07 \\
\hline & Gender & 0.35 & .07 & 0.60 & \\
\hline & AOD & 0.50 & .30 & $2.27 *$ & \\
\hline & AUDIT & 0.03 & .10 & 0.77 & \\
\hline \multirow[t]{5}{*}{ Step 2} & (Constant) & 1.07 & & 0.25 & .00 \\
\hline & Gender & 0.32 & .07 & 0.56 & \\
\hline & AOD & 0.50 & .30 & $2.26^{*}$ & \\
\hline & AUDIT & 0.30 & .09 & 0.66 & \\
\hline & FrSBe & 0.01 & .05 & 0.39 & \\
\hline \multirow[t]{6}{*}{ Step 3} & (Constant) & 7.17 & & 1.53 & $.09 * *$ \\
\hline & Gender & -0.07 & -.02 & -0.12 & \\
\hline & AOD & 0.27 & .16 & 1.16 & \\
\hline & AUDIT & 0.05 & .16 & 1.21 & \\
\hline & FrSBe & 0.04 & .30 & 1.95 & \\
\hline & BIS-11 & -0.09 & -.44 & $-2.55 * *$ & \\
\hline $\begin{array}{r}* \\
* *\end{array}$ & $\begin{array}{l}p<.05 \\
p=.01\end{array}$ & & & & \\
\hline
\end{tabular}

This is an Accepted Manuscript version of the following article:

Lyvers, M., Basch, V., Duff, H., \& Edwards, M. S. (2015). Trait impulsivity predicts D-KEFS Tower Test performance in university students. Applied Neuropsychology: Adult, 22(2), 88-93. https://doi.org/10.1080/23279095.2013.850693 